\title{
CYLINDRICITY OF ISOMETRIC IMMERSIONS INTO EUCLIDEAN SPACE
}

\author{
ROBERT MALTZ
}

ABSTRACT. A simple geometric proof is given for the Hartman-Nirenberg cylindricity theorem and some generalizations. Then the following cylino dricity theorem (unpublished) of S. Alexander is proved using the same idea.

Theorem. Let $f: M \rightarrow E^{n}$ be an isometric Euclidean immersion of the Riemannian product $M=M_{1} \times \ldots \times M_{k} \times E^{m}$ where the $M_{i}$ are not everywhere flat Riemannian manifolds, and $E^{m}$ denotes Euclidean m-space.

Then $C \geq k$, where $C$ denotes the codimension of the immersion; and if $C=$ $h$, then the immersion is cylindrical on the Euclidean factor.

1. Introduction. In this paper we prove some cylindricity theorems for isometric immersions of Riemannian manifolds in Euclidean space. These theorems all depend upon a remarkable lemma of P. Hartman. In its simplest form the lemma states that an isometric immersion of Euclidean 2-space $\mathbf{E}^{2}$ in $\mathbf{E}^{3}$, which maps a straight line in $\mathbf{E}^{2}$ onto a straight line in $\mathbf{E}^{3}$, must be cylindrical.

We first give a short and amusing geometrical proof of Hartman's lemma, which remarkably enough shows that smoothness assumptions on the immersion can be essentially dispensed with. The method used is completely general and may be of use elsewhere. In any case it leads to what must be the simplest possible proof of the well-known Hartman-Nirenherg cylindricity theorem. In fact, following Hartman, we state as Theorem 1 a partial generalization to Euclidean immersions of spaces of nonnegative Ricci curvature.

Finally, in $\$ 3$, we use Hartman's lemma to give a simple proof of an important unpublished cylindricity theorem of S. B. Alexander.

Although we have shown how to relax differentiability assumptions in the global part of these cylindricity theorems, we are not especially interested in that question here, so all maps and manifolds are assumed of class $C^{\infty}$ except where otherwise indicated.

2. The tetherball principle. In this section we formulate a completely general geometric principle and use it to prove the lemma below of P. Hartman [4]. The lemma will be used to obtain cylindricity theorems in this section and the following.

Received by the editors December 12, 1974.

AMS (11OS) subject classifications (1970). Primary 53A05, 53C40, 53C70.

Key words and phrases. Isometric immersions, Riemannian products, cylindricity. 
In order to state the lemma without differentiability assumptions, we will need the following definition. Let $M$ denote an arbitrary Riemannian manifold.

Definition. A map $f: M \rightarrow \mathbf{E}^{N}$ is a curve isometry if for every piecewise smooth curve $c$ in $M$, the image curve $f \circ c$ in $\mathbf{E}^{N}$ is rectifiable, and the length $L_{M}(c)$ of $c$ in $M$ equals the length $L_{E^{N}}(f \circ c)$ of $f \circ c$ in $\mathbf{E}^{N}$.

Remark. Curve isometry is obviously a generalization of Riemannian isometry (i.e. $f_{*}$ an isometry). However curve isometries are not necessarily differentiable (crease a piece of paper to obtain a counterexample: a nondifferentiable curve-isometric immersion of a portion of $\mathbf{E}^{2}$ into $\mathbf{E}^{3}$ ).

Now let $(r, s)$ and $\left(u_{1}, u_{2}, v\right)$ denote Cartesian coordinates in $\mathbf{E}^{2}$ and $\mathbf{E}^{3}$ respectively.

Lemma 1. Let $f: \mathbf{E}^{2} \rightarrow \mathbf{E}^{3}$ be a curve isometry mapping the entire saxis in $\mathbf{E}^{2}$ onto the v-axis in $\mathbf{E}^{3}$. Then $f(r, s)=\left(u_{1}(r), \dot{u}_{2}(r), s\right)$, i.e. the image $f\left(\mathbf{E}^{2}\right)$ is a cylinder $g \times V$, where $g$ denotes the plane curve $\left(u_{1}(r)\right.$, $\left.u_{2}(r)\right)$ and $V$ denotes the v-axis.

Remark. The differentiability of $f$ is clearly dependent only on the differentiability of the curve $g$. If, for instance, $g$ is piecewise differentiable, then the nondifferentiable points of $f$ consist of lines parallel to the $v$-axis (creases!). Intuitively, if such a crease were not parallel to the $s$ axis in $\mathbf{E}^{2}$ it would intersect that axis, contradicting an assumption in the lemma.

The proof is based on the following considerations. If an arbitrary Riemannian manifold $M$ is isometrically immersed in a Euclidean space $\mathbf{E}^{n}$ by a map $f$, then for any two points $p, q$ in $M$, we have

$$
d_{M}(p, q) \geq\|f(p)-f(q)\|
$$

where $d_{M}$ and \|\| denote, respectively, intrinsic Riemannian distance in $M$ and Euclidean distance in $E^{n}$. This inequality derives, of course, from the definition of $d_{M}$, and so persists if $f$ is only curve isometric in the sense of Lemma 1:

$$
\begin{aligned}
d_{M}(p, q) & =\inf _{c}\left\{L_{M}(c) \mid c \text { a curve in } M \text { joining } p \text { to } q\right\}=\underset{c}{\inf }\left\{L_{\mathrm{E}^{N}}(f \circ c)\right\} \\
& \geq \inf _{c}\left\{\left.L_{\mathrm{E}^{N}}(c)\right|_{c} \text { a curve in } \mathrm{E}^{N} \text { joining } p \text { to } q\right\} \\
& =\|f(p)-f(q)\| .
\end{aligned}
$$

We may now state the following principle.

The tetherball principle. Let $f: M \rightarrow \mathrm{E}^{n}$ be a curve isometry of a Riemannian manifold $M$ into $\mathbf{E}^{n}$. Then if $f(p)$ is known for some fixed $p$ in $M, f(q)$ must lie somewhere in the closed Euclidean ball of radius 
$d_{M}(p, q)$ with center $f(p)$, where $q$ is an arbitrary point in $M$. (Note that a tetherball, wherever it is located in space, must lie somewhere in a Euclidean ball of radius equal to the length of the tether rope with the top of the pole as center. Hence we refer to this principle as the tetherball principle.)

Proof of Lemma l. Consider an arbitrary point $q$ in $\mathbf{E}^{2}$ with coordinates $(r, s)$. We first show that $v(q)=s$. To do so, consider the point $p=(0, s+h)$. The distance from $p$ to $q$ in $\mathbf{E}^{2}$ is exactly $\left(h^{2}+r^{2}\right)^{1 / 2}$. Hence $f(q)$ must lie in the closed $\mathbf{E}^{3}$-ball $B_{h}$ of radius $\left(h^{2}+r^{2}\right)^{1 / 2}$ and center $f(p)=(0,0, s+h)$ by the tetherball principle. Similarly, using $p^{\prime}=(0, s-h)$, we find $f(p)$ lies some where in the closed $\mathbf{E}^{3}$-ball $B_{-h}$ of radius $\left(h^{2}+r^{2}\right)^{1 / 2}$ and center $(0,0, s-h)$. It follows immediately that $(s+h)-\left(r^{2}+h^{2}\right)^{1 / 2} \leq v(q) \leq(s-h)+$ $\left(r^{2}+h^{2}\right)^{1 / 2}$, and these inequalities hold for all $h$. As $h \rightarrow \infty$, we find $s \leq$ $v(q) \leq s$ as required. Geometrically, we have $f(q) \in B_{h} \cap B_{-h}$, a lensshaped region. As $h \rightarrow \infty$ these regions flatten out and tend in the limit to a disc of radius $r$ lying in the plane $v=s$ in $\mathbf{E}^{3}$. Hence, again $v(q)=s$.

Now let $q^{\prime}=(r, s+h)$. We know $\left\|f\left(q^{\prime}\right)-f(q)\right\| \leq h$, but $v\left(q^{\prime}\right)-v(q)=h$; it follows that $u_{i}\left(q^{\prime}\right)=u_{i}(q)$, or the $u_{i}$ are functions of $r$ alone.

Lemma 1 , as indicated by Hartman, can be extended in several ways. For one thing, the proof works equally well for $\mathbf{E}^{p}$ immersed in $\mathbf{E}^{q}, q \geq p$. We will also need the following generalization.

Lemma 2. Suppose $M=M_{1} \times \mathbf{E}^{1}$ is the Riemanmian product of a connected Riemannian manifold $M_{1}$ and $\mathbf{E}^{1}$, and suppose $f: M \rightarrow \mathbf{E}^{N}$ is an isometric immersion mapping a geodesic $g$ of form $\left(p_{1} \times \mathbf{E}^{1}\right)$ onto a straight line in $\mathbf{E}^{N}$. Then $f$ is cylindrical, i.e. there exists a decomposition $\mathbf{E}^{N}=\mathbf{E}^{N-1} \times \mathbf{E}^{1}$ such that $f=f_{1} \times I$ where $f_{1}: M_{1} \rightarrow \mathbf{E}^{N-1}$ is an isometric immersion and $I: \mathbf{E}^{1} \rightarrow \mathbf{E}^{1}$ is the identity immersion (equivalently, $\left.f\left(p_{1}, p_{2}\right)=\left(f_{1}\left(p_{1}\right), p_{2}\right)\right)$.

Proof. Join $p_{1}$ to a second point $q_{1}$ in $M_{1}$ by an arc $g_{1}$ in $M_{1}$. Now consider the two-dimensional submanifold $g_{1} \times g$ of $M$. The metric on $g_{1} \times g$ induced from $M$ must be the product metric, hence it must be the flat metric. It follows that we can apply an obvious extension of Lemma 1 to $g_{1} \times g$ immersed in $\mathbf{E}^{N}$, to obtain the result needed.

Lemma 1 and its generalizations can be used in combination with other results to obtain further cylindricity theorems. For instance, together with results of Chern-Kuiper [3] on index of relative nullity, the well-known Hartman-Nirenberg theorem on the cylindricity of Euclidean hypersurfaces in Euclidean space is immediately obtained (in fact, $(M-K)$-cylindricity is obtained for $\mathbf{E}^{M}$ immersed in $\left.\mathbf{E}^{M+K}, K<M\right)$. Lemma 1 makes it clear that dif ferentiability is needed only for the local part of the theorem. (Compare [5], [7], [9], and [10] for other approaches to this theorem.)

Again following Hartman [5], and using the recent generalization of the Toponogov splitting theorem to the case of nonnegative Ricci curvature (due 
to Cheeger-Gromoll [2]) we can state the following cylindricity theorem (see also Lichnerowicz [6] for a further generalization of the Cheeger-Gromoll result).

Theorem 1. Let $f: M \rightarrow \mathbf{E}^{N}$ be an isometric immersion of the complete Riemannian manifold $M$ in the Euclidean space $\mathbf{E}^{N}$. Assume further that $M$ has nonnegative Ricci curvature, and that $\nu>0$ everywhere, where $\nu$ denotes the index of relative nullity. Then $M=M_{1} \times \mathbf{E}^{n}$ and $f=f_{1} \times I$ where $f_{1}$ : $M_{1} \rightarrow \mathrm{E}^{N-n}$ and $I: \mathbf{E}^{n} \rightarrow \mathrm{E}^{n}$ is the identity immersion, and $n$ is the minimal value of $\nu$.

Proof. By well-known results on index of relative nullity (see, e.g., [1]), there exist $n$ linearly independent complete goedesics through some point in $M$ which are mapped onto straight lines in $\mathbf{E}^{N}$. By the CheegerGromoll-Toponogov result, $M=M_{1} \times \mathbf{E}^{n}$. The cylindricity of the immersion now follows by Lemma 2.

Remark. Professor Hartman has pointed out in a private communication that the tetherball principle is equally valid if $f: M \rightarrow \mathbf{E}^{N}$ is a curve contraction instead of an isometry (i.e. $f$ decreases length of curves).

3. Cylindricity of Riemannian product immersions. In this section we use Hartman's lemma to give a simple proof of an unpublished cylindricity theorem of S. B. Alexander, which complements results of J. D. Moore [8] and Alexander-Maltz [1]. Here $f$ nonflat means that $f$ has a nonzero sectional curvature.

Theorem 2. Let $f: M_{1} \times \ldots \times M_{k} \times \mathbf{E}^{M} \rightarrow \mathbf{E}^{N}$ be an isometric immersion, each $M_{i}$ a connected nonflat Riemannian manifold. Then the codimension $C$ of the immersion is $\geq k$; if $C=k$, then $f$ is cylindrical on the Euclidean factor $\mathbf{E}^{M}$.

Proof. We use the following inequality of $[1]: 0 \leq \mu(p)-\nu(p) \leq C_{-}$ $i(p)$, where $\mu$ and $\nu$ denote index of nullity and relative nullity, respectively, and where $i(p)$ denotes the number of factors $M_{i}$ with a nonzero sectional curvature at $p_{i}$, where $p=\left(p_{i}, \ldots, p_{k}, p_{k+1}\right)$.

So by assumption there exists some point $p$ at which $i(p)=k$, and $0 \leq$ $\mu(p)-\nu(p) \leq C-k$. Hence $C \geq k$.

If $C=k$, then $\mu(p)=\nu(p) \geq M$. In particular, every geodesic of form $\left(p_{1}, \ldots, p_{k}, g\right)$ where $g$ is a line in $\mathbf{E}^{M}$ is a nullity, and hence a relative nullity geodesic. As such it is mapped onto a straight line in $\mathrm{E}^{N}$ and the theorem follows immediately by Lemma 2, applied $M$ times.

Remark. When the codimension equals $k$ in Theorem 2, then not only is $f$ cylindrical, but it actually decomposes into a product of immersions of the individual factors $M_{i}$ and $\mathbf{E}^{M}$, provided the $M_{i}$ are compact [8] or satisfy Condition $(*)$ of [1]. 


\section{BIBLIOGR APHY}

1. S. Alexander and R. Maltz, Isometric immersions of Riemannian products in Euclidean space, J. Differential Geometry (to appear).

2. J. Cheeger and D. Gromoll, The splitting theorem for manifolds of nonnegative Ricci curvature, J. Differential Geometry 6 (1971/72), 119-128. MR 46 \#2597.

3. So S. Chern and N. H. Kuiper, Some theorems on the isometric imbedding of compact Riemann manifolds in euclidean space, Ann. of Math. (2) 56 (1952), 422430. MR 14, 408.

4. P. Hartman, On the isometric immersions in Euclidean space of manifolds with nonnegative sectional curvatures. II, Trans. Amer. Math. Soc. 147 (1970), 529540. MR 41 \#7586.

5. P. Hartman and L. Nirenberg, On spherical image maps whose Jacobians do not change sign, Amer. J. Math. 81 (1959), 901-920. MR 23 \# A4106.

6. A. Lichnerowicz, Variétés kähleriennes à première classe de Chern non negative et variétés riemanniennes à courbure de Ricci généralisée non negative, $\mathrm{J}$. Differential Geometry 6 (1971/72), 47-94. MR 45 \#9274.

7. W. S. Massey, Surfaces of Gaussian curvature zero in Euclidean 3-space, Tôhoku Math. J. (2) 14 (1962), 73-79. MR 25 \#2527.

8. J. D. Moore, Isometric immersions of riemannian products, J. Differential Geometry 5 (1971), 159-168. MR 46 \#6249.

9. K. Nomizu, On hypersurfaces satisfying a certain condition on the curvature tensor, Tôhoku Math. J. (2) 20 (1968), 46-59. MR 37 \#2138.

10. A. V. Pogorelov, Extensions of the theorem of Gauss on spherical representation to the case of surfaces of bounded extrinsic curvature, Dokl. Akad. Nauk SSSR 111 (1956), 945-947. (Russian) MR 19, 309.

NATIONAL RESEARCH INSTITUTE IN MATHEMATICAL SCIENCES, BOX 395, PRETORIA, SOUTH AFRICA 